
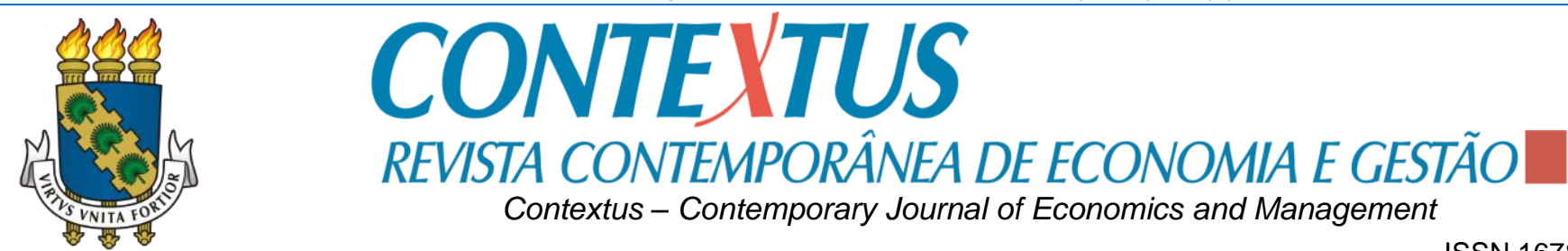

Contextus - Contemporary Journal of Economics and Management

ISSN 1678-2089

UNIVERSIDADE

ISSNe 2178-9258

FEDERAL do CEARá

www.periodicos.ufc.br/contextus

\title{
Fatores determinantes de sucesso: Uma análise dos projetos eólicos dos leilões de energia de reserva da ANEEL
}

\section{Success conditional features: An analysis of the wind power projects of reserve energy of ANEEL \\ Factores determinantes del éxito: Un análisis de los proyectos de viento de la subasta de reserva de ANEEL}

https://dx.doi.org/10.19094/contextus.2020.42457

\section{Antônio Vinícius Silva Caldas}

http://orcid.org/0000-0002-9980-5911 (iD Universidade Federal de Sergipe

Doutor em Administração

aulasdefinancas@gmail.com

\section{Antônio Francisco de Almeida da Silva Júnior}

http://orcid.org/0000-0002-4417-5991 (it)

Universidade Federal da Bahia

Doutor em Engenharia Aeronâutica e

Mecânica

afranc13@gmail.com

\section{Informações sobre o Artigo}

Submetido em 18/10/2019

Versão final em 08/01/2020

Aceito em 09/01/2020

Publicado online em 27/02/2020

Comitê Científico Interinstitucional

Editor-Chefe: Diego de Queiroz Machado Avaliado pelo sistema double blind review (SEER/OJS - versão 3)

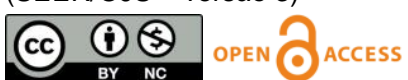

\section{RESUMO}

Este estudo estimou os fatores característicos de projetos de usinas eólicas bem-sucedidos, considerando exclusivamente os dados finais dos leilões de energia de reserva. Assim, foram analisados os resultados de 150 empreendimentos ganhadores dos certames ocorridos entre 2011 e 2015. Utilizou-se a ACP para identificação dos fatores que diferenciaram os projetos que se transformaram em usinas daqueles que fracassaram neste intento. Foram calculados os escores para cada projeto analisado, tendo como data de corte o mês de agosto de 2019. Dentre os projetos que tiveram maiores investimentos e se propuseram a gerar mais energia elétrica, $90,91 \%$ foram bem-sucedidos. Constatou-se ainda que os percentuais de deságios não tiveram uma relação significativa com o sucesso ou fracasso na transformação dos projetos em usinas.

Palavras-chave: Projetos de investimentos; Usinas eólicas; Leilões de energia de reserva; Análise dos componentes principais; Sucesso e fracasso.

\section{ABSTRACT}

This study estimated the characteristic features of successful wind power projects, considering only the final data of the reserve energy auctions. Thus, we analyzed the results of 150 projects that won the competition between 2011 and 2015. We used PCA to identify the features that differentiated the projects that became plants from those that failed in this regard. We calculated scores for each project analyzed, with a cut-off date of August 2019. Among the projects that had the largest investments and proposed to generate more electricity, $90.91 \%$ were successful. We found out that the percentage of negative goodwill did not have a significant relationship with the success or failure of turning projects into plants.

Keywords: Investment projects; Wind power plants; Reserve energy auctions; Principal component analysis; Success and failure.

\section{RESUMEN}

Este estudio estimó los factores característicos de los proyectos exitosos de parques eólicos, considerando solo los datos finales de las subastas de energía de reserva. Así, se analizaron los resultados de 150 proyectos que ganaron la competencia entre 2011 y 2015 . ACP se utilizó para identificar los factores que diferenciaron los proyectos que se convirtieron en plantas de aquellos que fallaron. Las puntuaciones se calcularon para cada proyecto analizado, con una fecha límite de agosto 2019. Entre los proyectos que tuvieron las mayores inversiones y propusieron generar más electricidad, el $90.91 \%$ tuvieron éxito. También se descubrió que el porcentaje de buena voluntad negativa no tenía una relación significativa con el éxito o el fracaso de convertir los proyectos en plantas.

Palabras clave: Proyectos de inversión; Plantas de energía eólica; Subastas de energía de reserva; Análisis de componentes principales; Éxito y fracaso.

\section{Como citar este artigo:}

Caldas, A. V. S, \& Silva, A. F. A., Jr. (2020). Fatores determinantes de sucesso: Uma análise dos projetos eólicos dos leilões de energia de reserva da ANEEL. Contextus - Revista Contemporânea de Economia e Gestão, 18(1), 1-13. https://dx.doi.org/10.19094/contextus.2020.42457 


\section{INTRODUÇÃO}

Nos dias atuais, a queima de carvão continua sendo a principal forma de produção de energia elétrica, causando danos ao planeta devido à emissão de gases de efeito estufa. Neste sentido, os esforços em busca de fontes de energias limpas encontram-se alicerçados nas necessidades de diminuir as agressões ao meio ambiente e de atender à crescente demanda oriunda da dinamicidade econômica. Como resultado, pela primeira vez em 40 anos, observou-se uma estabilização da emissão de dióxido de carbono. Diante desse cenário, o Brasil ganha destaque no uso das energias renováveis, principalmente as convencionais, como são o caso das hidroelétricas. Essas compõem $64 \%$ da matriz energética brasileira, o que deixa o país à mercê dos ciclos das chuvas. Um exemplo disso é que o valor dos contratos de energia e a quantidade de suprimento termoelétrico que pode ser adquirida pelas distribuidoras para cumprir suas obrigações são determinados por modelos matemáticos baseados nas estimativas pluviométricas para os próximos cinco anos (Bondarik, Pilatti, \& Horst, 2018; Campos, Ramos, \& Azevedo, 2016; González, Riba, Rius, \& Puig, 2015; Monteiro, Monteiro, Mariano, Urbanetz, \& Romano, 2017; Soares \& Campos, 2018).

Visando à necessidade de diversificação da matriz energética, o Brasil é um dos pioneiros na geração de energias limpas não convencionais, a exemplo da eólica e da fotovoltaica. Como meios de fomentar os investimentos nessas fontes de energia, o governo brasileiro faz uso de alguns mecanismos de dinamização, a exemplo do pagamento de tarifas prêmio (feed-in tariff), do incentivo fiscal, do financiamento público a taxas mais baixa e dos leilões de energia de reserva (LERs). Os LERs ocorrem em um ambiente de contratação regulada ( $A C R$ ) e são promovidos, direta ou indiretamente, pela Agência Nacional de Energia Elétrica (ANEEL) objetivando a contratação de energia por um preço mais acessível e atrair o interesse dos investidores, por meio da garantia na estabilidade das tarifas e da compra da energia gerada por um período de vinte anos (Carmo, Lima, Nunes, \& Saad, 2018; Vargas, Saavedra, Samper, Rivera, \& Rodriguez, 2016).

Segundo a ANEEL (2019), durante o período de 2011 a 2015, foram realizados cinco LERs que possibilitaram a contratação de 150 empreendimentos eólicos. De acordo com as informações do Operador Nacional do Sistema Elétrico (ONS) (2019), 31,33\% desses empreendimentos não conseguiram ser implementados, ou seja, não se concretizaram em usinas, extrapolando os prazos contratuais, o que os levou a ser considerados inviáveis. Oportuno destacar que esses empreendimentos foram descontratados, tiveram suas outorgas de autorização revogadas ou se encontravam paralisados por mais de 2 anos no momento da análise dos dados deste estudo.

Bayer, Berthold e Freitas (2018) consideram que um projeto de energia renovável pode fracassar em sua implementação devido aos seguintes motivos: problemas na rede de transmissão, descumprimento de prazos de entrega das matérias-primas por parte dos fornecedores, dificuldades de acesso a fontes de financiamentos, atrasos nas licenças ambientais, conflitos com o uso da terra, falta de experiência e/ou comportamento de risco dos investidores.

Del Rio e Linares (2014) e Kreiss, Ehrhart e Haufe (2017) atestam que os deságios elevados (underbidding) são as principais razões para que haja atrasos nos cronogramas de implantação dos projetos e ocorram as paralisações dos mesmos. Entretanto, os autores não deixam claro se menores percentuais de deságios podem motivar um maior sucesso na transformação dos empreendimentos em usinas. É oportuno salientar que, segundo dados da ANEEL (2019), os 150 projetos vencedores dos LERs foco deste estudo ofereceram deságios médios de $11,86 \%$ sobre o preço teto proposto nos certames.

Deste modo, pode-se perceber que, com exceção dos percentuais dos deságios, todos os fatores apontados pelos autores acima mencionados são alheios às informações que possam ser obtidas unicamente com base nos dados dos empreendimentos vencedores dos leilões de energia de reserva, a exemplo do valor do investimento a ser realizado, do megawatt hora a ser gerado e da volatilidade oriunda dos fluxos de caixa dos projetos.

Diante do exposto, levantou-se a seguinte questão norteadora: quais são os fatores, considerando-se exclusivamente os dados dos projetos vencedores dos leilões de energia de reserva ocorridos entre 2011 e 2015, que se mostraram mais determinantes para que os projetos de usinas eólicas tivessem mais possibilidades de se tornarem bem-sucedidos?

Este estudo objetiva estimar os fatores que se mostraram mais determinantes para que os projetos de usinas eólicas tivessem maiores chances de se tornarem bem-sucedidos, considerando exclusivamente os resultados finais dos leilões de energia de reserva ocorridos entre 2011 e 2015.

Neste sentido, foi levantada a seguinte hipótese: A dimensão de um projeto eólico é um fator determinante para o seu sucesso, inpendentemente do percentuaal de deságio oferecido. Como proxy da dimensão do projeto foram utilizadas as seguintes variáveis: geração de energia (MWh) e valor do investimento.

Vislumbra-se que este trabalho possa trazer como contribuição teórica e empírica a identificação das características que majoram as possibilidades de um projeto de usina eólica ser bem-sucedido, considerados os dados obtidos, exclusivamente, por meio dos projetos vencedores dos leilões de energia de reserva. Os resultados encontrados poderão servir como base na tomada de decisão de um investidor ao cogitar a possibilidade de participar de um certame da ANEEL, em complemento às abordagens de avaliação de projetos de investimento, a exemplo do valor presente líquido e da 
teoria das opções reais.

Além da presente introdução, este trabalho está dividido em quatro partes. A primeira traz o referencial teórico. A segunda apresenta os aspectos metodológicos. A terceira faz a análise e discussão dos dados. A quarta destina-se às considerações finais.

\section{REFERENCIAL TEÓRICO}

$\mathrm{Na}$ presente seção, são discutidas as energias renováveis no Brasil e as causas de fracasso na implementação de projetos já identificadas na teoria.

\subsection{As Energia Renováveis no Brasil}

As energias renováveis se apresentam como uma solução bastante viável para os problemas ambientais, principalmente para os associados à utilização de combustíveis fósseis, a exemplo do petróleo, gás mineral e o carvão, sendo esse último atualmente a principal forma de produção de energia elétrica, causando danos ao planeta devido à emissão de gases de efeito estufa (Agaton \& Karl, 2018).

Apesar de contar com fontes bastante renováveis, a matriz energética brasileira possui uma forte participação da energia hidráulica (Nascimento, 2017). Segundo dados de Sawin (2017), o Brasil é o segundo país que mais investe em hidroelétricas, só perdendo para a China. Esse tipo de fonte fornece mais de $60 \%$ da energia elétrica que é consumida no país, o que aumenta os riscos de falta de suprimento devido aos ciclos das chuvas e à possibilidade no aumento da demanda (Ribeiro \& Silva, 2016).

Neste sentido, existe a importância de diversificar o portfólio por meio das tecnológicas renováveis, a exemplo da eólica e da fotovoltaica, a fim de evitar a dependência exclusiva da água (Cuervo \& Botero, 2016).

O primeiro esforço no sentido de diversificar a matriz energética brasileira e minorar a sua a dependência dos recursos hídricos se deu por meio da Lei 10.438, de 26 de abril de 2002, que criou o Programa de Incentivo às Fontes Alternativas de Energia Elétrica (PROINFA) e determinou que as distribuidoras fossem obrigadas a comprar a energia gerada por essas fontes por um período de 15 anos (Zabaloy \& Guzowski, 2018). O PROINFA contemplou inicialmente as possibilidades de gerações eólicas, pequenas centrais hidroelétricas e biomassa. A energia fotovoltaica só foi abrangida por esse programa em 2013, visando à promoção da competitividade (Lei 12.783, 2013) .

A busca pela diversificação foi um dos objetivos da Empresa de Pesquisa Energética (EPE) (2017) ao elaborar o Plano Decenal de Expansão de Energia 2026, conforme é apresentado na Figura 1.

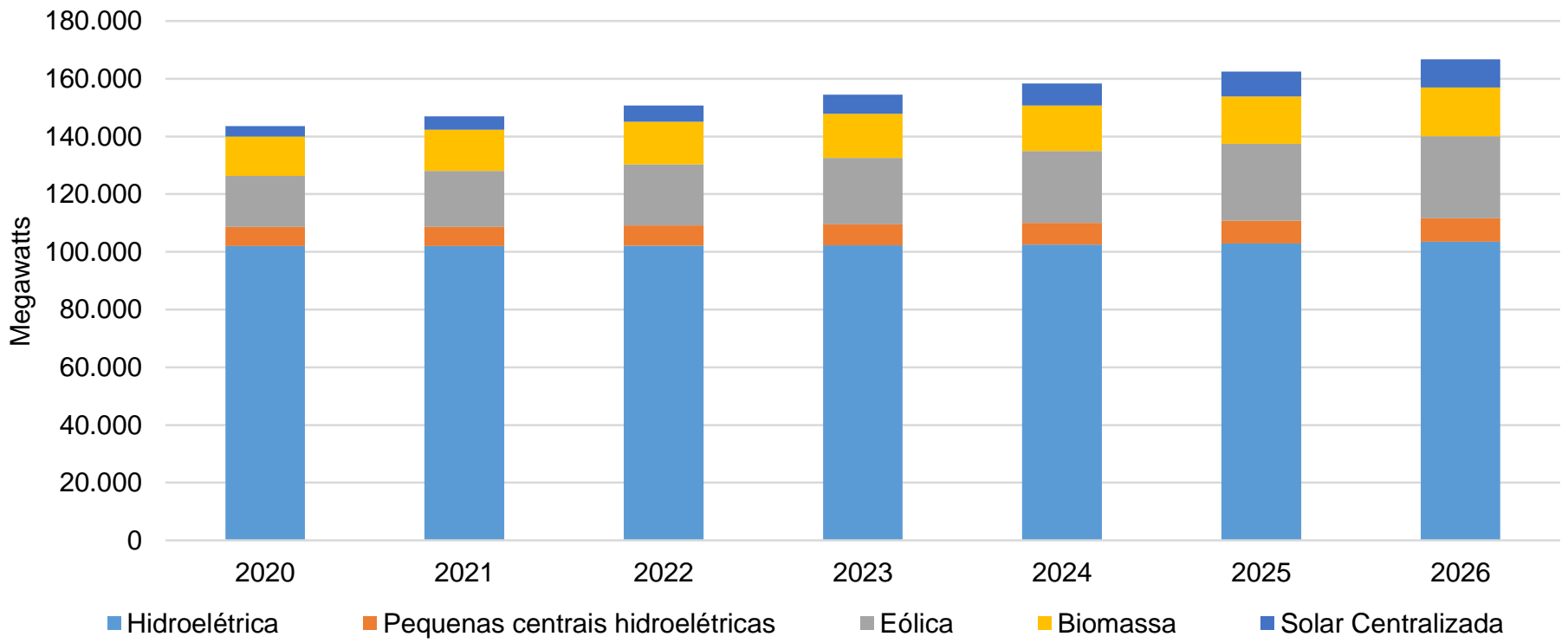

Figura 1. Evolução da capacidade instalada para o plano de expansão de referência. Fonte: elaborada com base em EPE (2017).

Na Figura 1, observa-se que, ao se comparar 2020 com 2026, é almejado um crescimento de $15,90 \%$ na capacidade instalada total das energias renováveis no plano de expansão de referência, saindo de um patamar de 145.568 para 168.716 megawatts. Neste período, esperase uma desaceleração no crescimento na capacidade instalada das fontes controláveis (hidroelétricas), que deverão ter um crescimento de apenas 1,43\%, 102.008 para 103.466 megawatts. No entanto, espera-se uma majoração mais do que proporcional nas fontes eólicas e solar centralizada, no montante de $61,35 \%$ e $163,93 \%$, respectivamente. Segundo Nakabayashi (2014), a energia solar centralizada é o tipo licitado nos LERs da ANEEL, está distante dos centros de carga e tem ganhos de escala, necessitando de grandes linhas de transmissão para que a energia possa ser transportada.

A Figura 2 apresenta como está planejada a participação das fontes de geração de energia no Plano 
Decenal de Expansão de Energia 2026. Por meio do Plano Decenal de Expansão de Energia 2026, pretende-se diminuir em $16,13 \%$ a participação das fontes de energia hidráulicas de um patamar de $62 \%$ para $52 \%$, considerando o período de 2020 a 2026. Em contrapartida, as outras renováveis (pequenas centrais hidroelétricas, eólicas, fotovoltaicas e de biomassa) têm uma previsão de crescimento de $25 \%$ no mesmo intervalo de tempo.

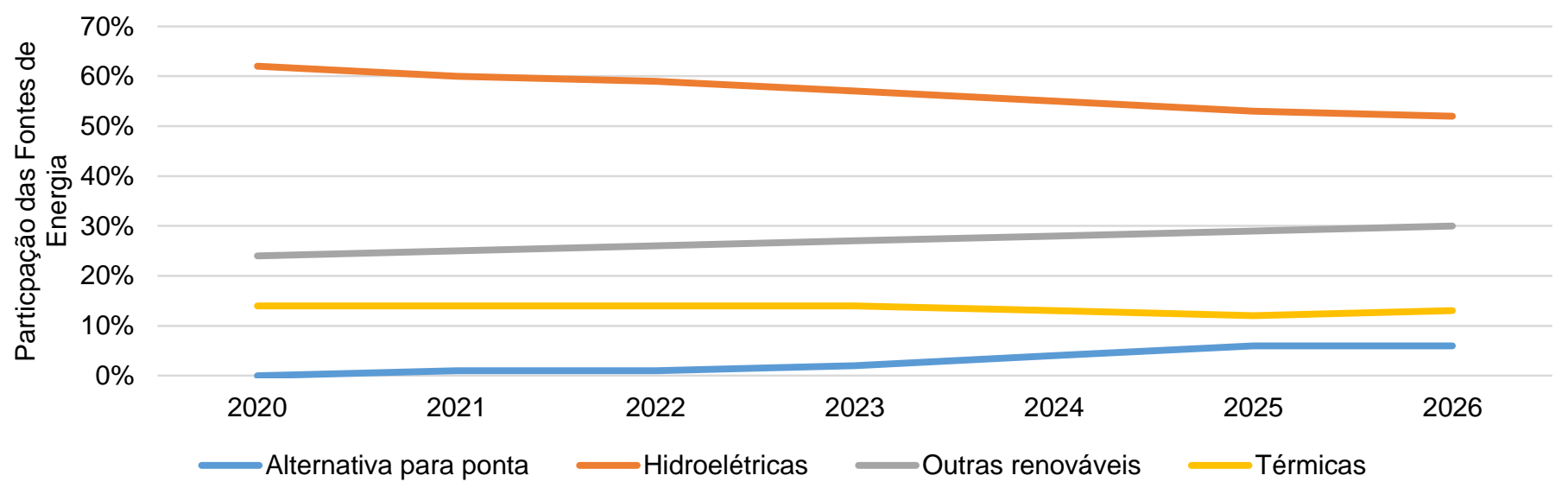

Figura 2. Participação das fontes de geração de energia.

Fonte: elaborada com base em EPE (2017).

De acordo com Sawin (2017), dos US\$ 279.8 bilhões investidos globalmente na geração deste tipo de energia, $38 \%$ foram destinados a fontes eólicas. Rodrigues, Perobell e Vasconcelos (2017) corrobaram essa visão e salientam que existe um aspecto de complementariedade entre a geração de energia eólica e as hidrelétricas, posto que os ventos mais fortes ocorrem nos períodos de menos chuvas.

A energia eólica vem conquistando espaço na matriz energética brasileira e seus custos são competitivos em relação às outras tecnologias tradicionais, tais como as termoelétricas, apesar de ainda carecer de uma maior potência e variabilidade em sua produção (EPE, 2017). Além disso, ela acarreta um impacto ambiental menor do que a fotovoltaica e outras formas de energia limpa (Torinelli, Silva, \& Andrade, 2018). É oportuno salientar que - Brasil é o maior mercado da América Latina na geração deste tipo de energia (Sawin, 2018).

Como uma forma de fomentar os investimentos em energias renováveis, o governo brasileiro vem utilizando a tarifa prêmio (Silva, Ribeiro, \& Quintella, 2018). Esse é um sistema no qual toda a energia injetada na rede é remunerada de forma a garantir uma taxa interna de retorno satisfatória para os investidores (Nascimento, 2017). Isso ocorre porque a tarifa prêmio tem um valor mais elevado do que o cobrado na rede pública, o que faz com que seja mais vantajoso vender toda a energia que foi gerada para a rede (Castro, 2015).

Outra maneira de dinamização encontrada pelo governo é o financiamento de projetos a uma taxa de juros mais baixa (Silva et al., 2018). Existe ainda o incentivo fiscal e o financiamento público para fomentar a geração de energias renováveis. Apesar do desafio, também é crescente o interesse privado em financiar esses tipos de empreendimentos (Torinelli et al., 2018). Por fim, destacase os leilões promovidos pela ANEEL que, apenas durante o período de 2011 a 2015, possibilitaram a contratação de 150 empreendimentos eólicos (ANEEL, 2019).

Os leilões são uma espécie de licitação por meio da qual ocorrem as contratações dos projetos de geração e transmissão de energia elétrica, sendo compostos por três grandes grupos de energia: nova, existente e reserva. Esses grupos são subclasificados em A-1, A-3 e A-5, sendo que esses números representam os prazos nos quais os projetos precisam entrar em funcionamento depois da realização dos certames (Ribeiro \& Silva, 2016).

Em 2005, foi lançado o primeiro leilão de energias renováveis. Entretanto, além da água, a única fonte limpa contemplada por esse certame foi a biomassa (Ferreira, Patah, \& Faria 2017). A inclusão das gerações eólica e fotovoltaica, segundo dados da ANEEL (2019), só se deu com o surgimento dos LERs que ocorreram em 2009 e 2014, respectivamente. Os leilões de 2009 tiveram um preço médio de $R \$ 148,39 / \mathrm{MWh}$ e serviram como modelo para os demais certames do setor elétrico (Pinto, Martins, \& Pereira, 2017).

De acordo com a Câmara de Comercialização de Energia Elétrica (CCEE) (2015), a energia de reserva é adicionada às ofertas das usinas concedidas e autorizadas, após ser sido verificada a garantia de suprimento do Sistema Integrado Nacional (SIN), e não pode ser revendida após a compra.

O Decreto 6.353, de 16 de janeiro de 2008, trata a energia de reserva da seguinte forma:

A energia de reserva será contratada mediante leilões a serem promovidos pela ANEEL, direta ou indiretamente, conforme as diretrizes do Ministério de Minas e Energia (MME). Entende-se por energia de reserva aquela destinada a aumentar a segurança no fornecimento de energia elétrica ao SIN, proveniente de usinas especialmente contratadas para este fim (Decreto 6.353, 2008). 
Entretanto, mesmo com todo esforço em prol da expansão das energias renováveis, em agosto de 2019 dados do ONS (2019) atestavam que havia uma taxa de fracasso de $31,33 \%$ na implantação dos 150 projetos eólicos contratados por meio dos cinco LERs ocorridos entre 2011 e 2015.

\subsection{Causas de fracassos na implementação dos projetos identificadas na literatura}

De acordo com Bradshaw (2018), os estudos devem contemplar uma avaliação ex-post nos leilões de energia, tendo em vista que eles só poderão ser considerados efetivamente bem-sucedidos se os projetos por eles contratados forem concretizados em usinas. Alguns estudos têm se debruçado sobre a questão dos fatores que podem levar um projeto de energia renovável ao fracasso. Del Rio e Linares (2014) defendem os percentuais elevados de deságio (underbidding) como um dos principais responsáveis pelos atrasos nos cronogramas de implantação das usinas. Ratificando essa ideia, Kreiss, Ehrhart e Haufe (2017) atestam que o underbidding é um dos principais riscos de um projeto não ser implementado, ou seja, a usina não ser construída. Viana (2018) esclarece que a não construção da usina está mais relacionada com a capacidade econômica do empreendedor. Para que isso não aconteça, Atalay, Kalfagianni e Pattberg (2017) salientam a necessidade de aplicação de penalidades que, no caso extremo, podem ocasionar a revogação da outorga de autorização do empreendimento.

Ferreira et al. (2017) destacam a assimetria de informação entre os concorrentes dos LERs, principalmente no tocante ao montante de energia a ser comercializado e às idiossincrasias das fontes eólicas e fotovoltaicas. Ratificando essa assertiva, Hochberg e Poundneh (2018) destacam que as empresas com mais experiências em leilões têm maiores chances de serem bem-sucedidas.

Bayer et al. (2018) alertam que a falta de conexão à rede elétrica e os problemas com fornecedores são os principais motivos para os atrasos nas entregas das usinas. Os autores destacam também, mas com menor importância, o financiamento do projeto, licenças ambientais, logística e falhas no gerenciamento como outras razões para o atraso ou mesmo o fracasso de um projeto ser efetivado em usina. Nesta mesma linha, Viana (2018) acrescenta os trâmites administrativos e os problemas financeiros como outras razões para atrasos nos projetos.

\section{METODOLOGIA}

Este estudo exploratório e quantitativo objetivou estimar, por meio da técnica da análise dos componentes principais (ACP) e da regressão, os fatores que se mostraram mais determinantes para que os projetos de usinas eólicas tivessem maiores chances de se tornarem bem-sucedidos, considerando exclusivamente os resultados finais dos LERs ocorridos entre 2011 e 2015. Os empreendimentos que entraram em atividade comercial até agosto de 2019, data de corte utilizada neste estudo, foram considerados bem-sucedidos. Os demais não conseguiram ser implementados, ou seja, não se concretizaram em usinas, extrapolando os prazos contratuais. Oportuno destacar que esses empreendimentos foram descontratados, tiveram suas outorgas de autorização revogadas ou se encontravam paralisados por mais de 2 anos no momento da análise dos dados deste estudo.

O motivo da escolha da ACP neste estudo foi a sua capacidade de fazer a junção de elementos com base na variação de suas características. Por meio da ACP, é possível verificar relações que não estavam muito claras entre as variáveis, o que amplia a capacidade de interpretação do conjunto observado (Oliveira \& Oliveira, 2017).

A ACP é uma técnica estatística multivariada utilizada para redução da dimensionalidade de um conjunto de dados, de forma que a sua variabilidade seja preservada o máximo possível. Logo, os dados originais são dispostos em uma matriz de rotação, ou seja, transformados linearmente em um outro conjunto menor de variáveis escolhidas entre aquelas com a maior variância, de forma a capturar a essência dos dados originais, o que possibilita a análise conjunta de diversos fatores. Cada combinação linear corresponde a uma componente principal cujo número total é igual à quantidade das variáveis utilizadas. A primeira componente principal é aquela que representa a maior variância de dados, ao passo que a segunda é perpendicular à primeira e abrange a segunda maior variância, e assim sucessivamente até que se chegue à última componente (Hongyu, Sandanielo, \& Oliveira, 2016; Jolliffe \& Cadima, 2016).

Foram utilizadas as seguintes variáveis para a realização da ACP deste estudo: percentual de deságio (DES), investimento (INV), valor presente do fluxo de caixa livre (VP) e megawatts-hora (MWh). Essas variáveis foram as que se obteve a partir dos dados disponibilizados nos projetos de energia eólica vencedores dos certames da ANEEL. Por esse motivo, questões alheias aos dados inerentes aos resultados dos LERs não foram consideradas neste estudo.

É oportuno salientar que a desconsideração da volatilidade das opções reais como uma das variáveis que poderiam ser extraídas dos fluxos de caixa dos projetos é justificada pelo método escolhido para a sua estimação. $\mathrm{Na}$ visão de Pereira (2019), a metodologia mais adequada é a proposta por Brandão, Dyer e Hahn (2012). Segundo esses autores, a volatilidade depende das incertezas às quais os empreendimentos estejam sujeitos e aos pesos dos seus respectivos custos fixos. Por se tratarem de projetos relativos à mesma fonte de energia, enfrentando as mesmas incertezas e com os mesmos percentuais de custo fixos, o valor da volatilidade seria igual para todos eles. $\mathrm{Na}$ visão de Rossi (2017), quando uma variável qualquer tem variância nula, ela deve ser desconsiderada da ACP. 
Para verificar se as variáveis selecionadas poderiam ser aplicadas na ACP, Lee (2014) recomenda a utilização prévia do teste de Kaiser-Meyer-Olkin (KMO) e da esfericidade de Bartlett. Para Kaiser (1974), o KMO desejável é o superior a 0,7 , podendo ser aceitas variáveis que gerem um KMO superior a 0,5. Hair, Black, Babin e Anderson (2010) esclarecem que a hipótese nula do teste de Bartlett ( $\mathrm{HO}=$ variáveis não são intercorrelacionadas) deve ser refutada ( $p$-valor $<5 \%$ de significância). Para os autores, refutar a $\mathrm{HO}$ indica que, pelo menos, algumas das variáveis devem ser correlacionadas.

Na visão de Ketsiri (2012), é justificado o processo de redução das variáveis inicialmente escolhidas em uma pesquisa quando um dos testes aplicados (KMO e Bartlett) aponta para tal necessidade. Logo, um KMO menor do que 0,5 indica que, possivelmente, o somatório das correlações parciais é superior à soma das correlações totais, ou seja, há uma associação maior da variável analisada isoladamente do que em conjunto com as demais, o que enseja a impropriedade do uso da mesma em uma ACP. Assim, quanto menor foi o valor do $\mathrm{KMO}$, mas fraca será a correlação entre as variáveis. Outrossim, um teste de Bartlett cujo p-valor seja maior do que $5 \%$ indica que existe uma relativa compactação nos padrões das correlações e que a ACP não produziria valores confiáveis.

Para a escolha da quantidade das componentes principais a serem retidas na ACP, utilizou-se o critério de Kaiser. Segundo Naik (2017), esse critério indica que só são relevantes as componentes principais que tenham os autovalores (eigenvalue) maiores do que 1, por apresentarem as maiores variâncias. Foram considerados como determinantes os autovetores (eigenvector) cujas cargas se apresentaram superiores a 0,5 em módulo, conforme é orientado por Lai, Cheng e Yeung (2004). Maskey, Fei e Nguyen (2018) ratificam essa informação e esclarecem ainda que o sinal de um autovetor (positivo ou negativo) pode ser interpretado da mesma forma que uma correlação, ou seja, indica se ele está associado direta ou indiretamente com os demais.

É oportuno destacar que a matriz de rotação gerada na ACP é resolvida por meio de uma equação de autovetores e autovalores. Por autovetores se entende o resultado da multiplicação da matriz de rotação por uma matriz coluna (vetor) cujo resultado é um múltiplo desse mesmo vetor. Já os autovalores podem ser entendidos como a máxima variância que se busca nos dados originais para cada componente principal. Quanto maior o autovalor, maior o autovetor. Assim, na primeira componente principal se encontram o par dos maiores autovetores e autovalores. Outrossim, a segunda componente expressa o segundo maior par, e assim por diante (Jolliffe \& Cadima, 2016).

Após a escolha das componentes principais mais relevantes, as mesmas foram utilizadas como regressoras em uma regressão logística, cuja variável dependente foi a situação de sucesso (1) ou fracasso (0) apresentada por cada projeto analisado. A regressão logística é a mais simples forma de agrupamento supervisionado, encontrando ampla aplicação em modelos dicotômicos (Kim, Chi, \& Kim, 2018; Spratt, Ju, \& Brasier, 2013). Na visão de Hair et al. (2010), trata-se de uma combinação da regressão múltipla com a análise discriminante. Entretanto, diferentemente da regressão múltipla, a variável dependente da logística é não-métrica. Além disso, o fato de poder aceitar diferentes tipos de preditores, não necessariamente distribuídos normalmente, é o que a diferencia da análise discriminante. Neste ponto, cabe ressaltar que apesar de Khanna, Sahu, Baths e Deshpande (2015) afirmarem que a regressão logística só admite regressores binários, diversos autores, a exemplo de Hair et al. (2010), Kuhn e Johnson (2013) e Sur e Candès (2019), esclarecem que esses podem ser métricos e/ou não métricos, devendo-se proceder à padronização dos mesmos, caso tenham dimensões diferentes.

O método de classificação utilizado para proceder à regressão logística foi o Logit. De acordo com Klieštik, Kočišová e Mišanková (2015), essa é a função normalmente empregada na predição da probabilidade de um evento ocorrer ou não. Corroborando essa assertiva, Bello, Oguntolu, Adetutu e Ojedokun (2016) esclarecem que função Logit pode ser entendida como o logaritmo natural da razão das probabilidades de ocorrência da variável dependente.

Quanto à coleta de dados, este estudo realizou uma pesquisa documental, ou seja, fez uso de fontes secundárias, conforme é ensinado por Lakatos e Marconi (2010). Foram utilizados os resultados dos LERs da ANEEL, considerados de uso público e disponíveis no website desse órgão regulador, no qual foram identificados os projetos vencedores dos certames ocorridos entre 2011 e 2015, além dos valores dos investimentos, dos megawatts-hora $(\mathrm{MWh})$ e dos preços regulados para cálculo da receita do fluxo de caixa e do percentual de deságio de cada projeto.

Durante a modelagem do fluxo de caixa livre, algumas premissas foram assumidas. $O$ seu valor presente (VP) foi calculado tradicionalmente, trazendo as entradas de líquidas para o momento zero através de uma taxa de desconto equivalente ao custo de capital próprio. De acordo com a legislação da ANEEL, todos os projetos estão submetidos a um percentual de $12,34 \%$ a.a. Esse normativo estabelece ainda que as estruturas de capital de todos os projetos são divididas igualmente entre recursos próprios e de terceiros, estando esses últimos submetidos a uma taxa de juros de $9,81 \%$ a.a. Por fim, considerou-se uma taxa livre de risco de 4,59\% a.a (Resolução Normativa n. 608, 2014). Com relação aos preços contratados, os mesmos sofreram atualizações anuais pelo Índice de Preços ao Consumidor Amplo (IPCA), nos 20 anos do contrato (Resolução Normativa n. 780, 2017). Esses preços foram multiplicados pela produção anual, sendo essa diminuída pelas perdas técnicas, sendo de $2,5 \%$ ao ano (Lindemeyer, 2018). Foram considerados custos de manutenção de $2,0 \%$ ao ano sobre o valor do investimento, conforme é sugerido por Fontanet (2012). Como base na Lei 11.488, de 15 de junho de 2007 
e nos convênios firmados pelo Conselho Nacional de Política Fazendária (CONFAZ), a exemplo do Convênio ICMS 109, de 21 de outubro de 2014, foram desconsideradas as existências de tributos incidentes sobre a receita e sobre o lucro.

Por meio das informações constantes nos atlas brasileiros de energia eólica, foram consideradas as variações médias da velocidade do vento (14,25\%), conforme está disposto em Amarante, Brower e Zack. (2012). Assaf (2019) sugere a utilização de um percentual de dividendos médio de $4 \%$ a.a. Por fim, os dados sobre as efetivas entradas em operação dos projetos de usinas eólicas estão disponíveis no website do ANEEL.

Para utilização da ACP, conforme é orientado por Jolliffe e Cadima (2016), as variáveis DES, INV, VP e MWh foram padronizadas a fim de corrigir as diferenças nas escalas, garantir que elas tivessem o mesmo peso e evitar a distorção no cálculo das variâncias. Para tanto, subtraiuse cada variável de sua respectiva média e dividiu-se o resultado pelo desvio padrão.

A variável objetivo (dependente), que representa a situação da empresa de ter entrado (1) ou não (0) em atividade, não foi considerada na ACP por essa não ser um algoritmo supervisionado.

Utilizou-se a planilha Excel 2016 para o tratamento das variáveis. Para a realização da ACP e da regressão logística, fez-se uso do software estatístico STATA versão 16.

\section{ANÁLISE E DISCUSSÃO DOS RESULTADOS}

Nesta seção são apresentadas as situações dos projetos das usinas eólicas por estado e determinados os fatores que se mostraram mais determinantes para que esses empreendimentos se tornarem bem-sucedidos, considerando exclusivamente os resultados finais dos LERs ocorridos entre 2011 e 2015.

\subsection{Situações dos projetos das usinas eólicas por estado}

A Tabela 1 apresenta as situações dos projetos de usinas eólicas vencedores dos LERs ocorridos entre 2011 e 2015. As situações 0 e 1 indicam, respectivamente, a situação de fracasso e de sucesso, considerando-se esse último como o cenário no qual os projetos foram efetivados em usinas que conseguiram entrar em atividade comercial até agosto de 2019.

Nessa data, os projetos considerados fracassados estavam descontratados, tiveram suas outorgas de autorização revogadas ou se encontravam paralisados por mais de 2 anos no momento da análise dos dados deste estudo.

\section{Tabela 1}

Situação dos projetos de usinas eólicas

\begin{tabular}{|c|c|c|c|c|c|c|c|c|}
\hline \multirow{2}{*}{ Situação } & \multicolumn{7}{|c|}{ Estados } & \multirow{2}{*}{ Total } \\
\hline & BA & $\mathrm{CE}$ & MA & $\mathrm{PE}$ & $\mathrm{PI}$ & $\mathrm{RN}$ & RS & \\
\hline 0 & 20 & 12 & 0 & 0 & 0 & 11 & 4 & 47 \\
\hline 1 & 49 & 1 & 1 & 10 & 17 & 20 & 5 & 103 \\
\hline Total & 69 & 13 & 1 & 10 & 17 & 31 & 9 & 150 \\
\hline
\end{tabular}

Fonte: elaboração própria.

Dos 150 projetos vencedores dos LERs, 47 deixaram de gerar energia eólica, o que significa uma taxa global de fracasso de $31,33 \%$. O principal estado na geração de energia foi a Bahia, que se propôs a sediar de $46 \%$ dos projetos ganhadores. Dos 69 empreendimentos, o citado estado teve sucesso na implementação de 49 , o que significa uma taxa de sucesso de $71,01 \%$.

Proporcionalmente ao número de projetos que receberam, os estados de Pernambuco, Piauí e Maranhão foram os que obtiveram mais êxito, alcançando a taxa de sucesso de $100 \%$. Antagonicamente, o Ceará teve uma taxa de fracasso de $92,31 \%$ posto que dos 13 projetos recebidos, apenas um foi capaz de gerar energia. Os estados do Rio Grande do Norte e do Rio Grande do Sul apresentaram taxas de fracasso de $35,48 \%$ e $44,44 \%$, respectivamente.

\subsection{Os fatores que se mostraram mais determinantes para o sucesso dos projetos de usinas eólicas}

Os possíveis fatores determinantes para o sucesso nos projetos de usinas eólicas foram estimados por meio da ACP, tendo os seguintes atributos comuns a todos os projetos: percentual de deságio (DES), investimento (INV), valor presente do fluxo de caixa livre (VP) e megawatts-hora (MWh). Todas essas variáveis foram calculadas a partir dos dados disponibilizadas nos LERs da ANEEL. Inicialmente foram realizados os testes de esfericidade de Bartlett e da adequação da amostra de KMO, conforme a Tabela 2.

\section{Tabela 2}

Testes de Bartlett e KMO

\begin{tabular}{c|c}
\hline Teste & Valores \\
\hline Esfericidade de Bartlett (p-valor) & 0,000 \\
\hline KMO & 0,562 \\
\hline
\end{tabular}

Fonte: elaboração própria.

Têm-se um p-valor não significativo estatisticamente a $5 \%$, o que refuta a hipótese nula de que as variáveis não são intercorrelacionadas. Logo, as variáveis escolhidas são colineares e não oriundas de uma matriz de identidade. $O$ teste KMO de 0,562 atende ao sugerido por Kaiser (1974) para adequação das variáveis, tornando as mesmas válidas para serem aplicadas na ACP. Desse modo, procedeu-se aos cálculos dos autovalores e os autovetores das componentes principais, conforme as Tabelas 3 e 4. 
Tabela 3

Cálculo dos autovalores

\begin{tabular}{c|c|c|c|c}
\hline Comp. & Autovalores & Diferença & Proporção & Acum. \\
\hline Comp1 & 2,32534 & 1,19533 & $58,13 \%$ & $58,13 \%$ \\
\hline Comp2 & 1,13001 & 0,75149 & $28,25 \%$ & $86,38 \%$ \\
\hline Comp3 & 0,37852 & 0,21239 & $9,46 \%$ & $95,85 \%$ \\
\hline Comp4 & 0,16613 &. & $4,15 \%$ & $100,00 \%$ \\
\hline
\end{tabular}

Fonte: elaboração própria.

\section{Tabela 4}

Cálculo dos autovetores

\begin{tabular}{c|c|c|c|c|c}
\hline Variáveis & Comp1 & Comp2 & Comp3 & Comp4 & $\begin{array}{c}\text { Não } \\
\text { explicado }\end{array}$ \\
\hline DES & $-0,0462$ & 0,9158 & 0,3138 & 0,2464 & 0 \\
\hline INV & 0,5884 & 0,0947 & $-0,6047$ & 0,5283 & 0 \\
\hline VP & 0,5417 & $-0,3004$ & 0,7307 & 0,2870 & 0 \\
\hline MWh & 0,5986 & 0,2493 & $-0,0426$ & $-0,7601$ & 0 \\
\hline
\end{tabular}

Fonte: elaboração própria.

Com pode ser visto na Tabela 3, das quatro componentes principais geradas na $A C P$, duas atenderam ao critério de Kaiser, ou seja, apresentaram autovalores superiores a 1. Essas componentes conseguiram juntas captar $86,38 \%$ da variância dos dados originais. Desde modo, as componentes 3 e 4 foram descartadas.

$\mathrm{Na}$ Tabela 4, a componente principal 1 (Comp1) mostra que o deságio é a única variável que tem uma carga pouco significativa (menor que 0,5 em módulo) para explicar a variância conjunta dos dados. Os atributos MWh, INV e VP foram os que apresentaram as maiores capacidade de explicação da variância, com cargas de 0,5986, 0,5884 e 0,5417, respectivamente. Assim, os projetos eólicos que possuíam essas três variáveis com valores mais elevados foram aqueles que mais se diferenciaram dentre os analisados. Por isso, denominouse a Comp1 de "Maiores Gerações e Maiores RiquezasMGMR".

Com relação à segunda componente principal (Comp2), apenas o atributo deságio merece destaque na Tabela 4, com uma carga de 0,9158. As demais tiveram pouca significância na explicação da variância total. Deste modo, denominou-se a Comp2 de "Maiores Deságios MDES".

Os escores das componentes MGMR e MDES podem ser encontrados a partir da ponderação de cada variável por suas respectivas cargas de autovetores, conforme está disposto nas equações (1) e (2).

MGMR $=-0,0462 \times$ DES $+0,5884 \times \mathrm{INV}+0,5417 \times \mathrm{VP}+$ $0,5986 \times \mathrm{MWh}$

MDES $=0,9158 \times$ DES $+0,0947 \times$ INV $-0,3004 \times$ VP + $0,2493 \times \mathrm{MWh}$

Como as variáveis que nominaram a componente MGMR foram positivas, os projetos de usinas com os escores positivos tendem a ter mais possibilidades de sucesso, conforme a Tabela 5.
Tabela 5

Aplicação da MGMR nos projetos eólicos analisados

\begin{tabular}{c|c|c|c|c|c}
\hline Escores & Proj. & $\begin{array}{c}\text { Não } \\
\text { Gerou }\end{array}$ & Gerou & $\begin{array}{c}\text { Taxa de } \\
\text { Fracasso }\end{array}$ & $\begin{array}{c}\text { Taxa de } \\
\text { Sucesso }\end{array}$ \\
\hline Positivos & 88 & 8 & 80 & $9,09 \%$ & $90,91 \%$ \\
\hline Negativos & 62 & 40 & 22 & $64,52 \%$ & $35,48 \%$ \\
\hline
\end{tabular}

Fonte: elaboração própria.

$\mathrm{Na}$ Tabela 5, pode ser observado que 88 projetos tiveram escores positivos de MGMR, sendo que desses 90,91\% conseguiram gerar energia eólica. Dos 62 restantes, apenas 22 entraram em atividade comercial, ou seja, uma taxa de fracasso de $64,52 \%$. Deste modo, podese afirmar que, em sua ampla maioria, os projetos de usinas eólicas bem-sucedidos foram aqueles destinados a um maior volume de geração de energia, que realizaram investimentos mais elevados e que se mostram mais capazes de gerar maiores fluxo de caixa livre. Os demais, por terem se comprometido a gerar menos $\mathrm{MWh}$, realizaram investimentos mais modestos, e, consequentemente, os fluxos de caixa livre obtidos foram menores, o que causou uma taxa de sucesso de apenas de $35,48 \%$.

No tocante à componente MDES, o principal atributo que a compõem é positivo. Assim, os projetos eólicos foram segregados de acordo com os escores obtidos, conforme é apresentado na Tabela 6 . Foram incluídos os valores dos atrasos médios a fim de verificar a consonância com o disposto na teoria, ou seja, percentuais de deságios mais elevados sendo motivadores de maiores atrasos nas implementações dos projetos, conforme afirmado por por Azuela e Barroso (2012) e Del Rio e Linares (2014).

\section{Tabela 6}

Aplicação da MDES nos projetos eólicos analisados

\begin{tabular}{c|c|c|c|c|c|c}
\hline Escores & Proj. & $\begin{array}{c}\text { Não } \\
\text { Gerou }\end{array}$ & Gerou & $\begin{array}{c}\text { Atraso } \\
\text { Médio }\end{array}$ & $\begin{array}{c}\text { Taxa de } \\
\text { Fracasso }\end{array}$ & $\begin{array}{c}\text { Taxa de } \\
\text { Sucesso }\end{array}$ \\
\hline Pos. & 45 & 15 & 30 & 2,55 & $33,33 \%$ & $66,67 \%$ \\
\hline Neg. & 105 & 33 & 72 & 1,41 & $31,43 \%$ & $68,57 \%$ \\
\hline
\end{tabular}

Fonte: elaboração própria.

A Tabela 6 mostra que 105 projetos ofereceram percentuais de deságios menores que a média geral, posto que apresentaram escores negativos da componente MDES. Observa-se ainda que o atraso médio desses empreendimentos foi de 1,41 anos, ou seja, um ano e cinco meses a mais do que determinado no contrato de energia de reserva. Por outro lado, 45 projetos obtiveram valores positivos da MDES, o que significa que os preços finais tiveram deságios maiores do que a média. Para esses empreendimentos, o atraso médio ficou em 2,55 anos, o que representa dois anos e sete meses de atraso a mais do que o acordado. Logo, a componente MDES está em harmonia com o disposto na literatura, tendo em vista ratificar que maiores percentuais de deságios proporcionam maiores atrasos nas implementações dos projetos eólicos. Entretanto, a MDES não parece responder bem a possibilidade de um empreendimento ser bem-sucedido, 
tendo em vista que, independentemente dos escores que os projetos tenham atingido, as taxas de sucesso e fracasso apresentadas foram praticamente as mesmas.

A fim de se verificar como as componentes MGMR e MDES se relacionaram com as realidades apresentadas por cada projeto em agosto de 2019, foi realizada uma regressão logística, tendo como variável dependente a transformação dos projetos em usinas, ou seja, as suas entradas ( 1 - sucesso) ou não (0 - fracasso) em atividade comercial. Os resultados da regressão são apresentados na Tabela 7.

\section{Tabela 7}

Regressão logística com as preditoras MGMR e MDES

\begin{tabular}{c|c|c|c|c|cc}
\hline Regressor & Coef. & $\begin{array}{c}\text { Erro } \\
\text { Padrão }\end{array}$ & $\mathrm{Z}$ & $\mathrm{P}>\mathrm{Z}$ & \multicolumn{2}{|c}{$\begin{array}{c}\text { Intervalo de } \\
\text { confiança (95\%) }\end{array}$} \\
\hline MGMR & 1,353 & 0,219 & 6,17 & 0,000 & 0,9236 & 1,7829 \\
\hline MDES & $-0,203$ & 0,215 & $-0,95$ & 0,344 & $-0,6245$ & 0,2177 \\
\hline Const. & 1,229 & 0,265 & 4,63 & 0,000 & 0,7088 & 1,7491 \\
\hline
\end{tabular}

Fonte: elaboração própria.

Foram necessárias cinco interações para estimar a regressão apresentada na Tabela 7. O pseudo $R^{2}\left(R^{2}\right.$ de McFadden) apresentou um valor de 0,3872. Segundo McFadden (1977), valores entre 0,2 e 0,4 significam uma boa qualidade de ajustamento. Pôde-se constatar que apenas a preditora MGMR foi estatisticamente significativa, tendo em vista o seu $p$-valor $(P>Z)$ inferior a $5 \%$. O teste $Z$ corrobora essa assertiva, o que indica que a MGMR afeta positivamente a probabilidade de o projeto de usina eólica ser bem-sucedido.

Com relação ao componente MDES, os testes apontam para a sua irrelevância na determinação de sucesso ou fracasso do projeto $(\mathrm{p}$-valor $=0,344$ e $Z=$ $0,95)$, o que está em consonância com o percebido na Tabela 6.

Diante do exposto, não se pode refutar a hipótese levantada neste estudo de que a dimensão do projeto eólico (geração de energia e valor do investimento) é um fator determinante para que os mesmos se transformem efetivamente em usinas, inpendentemente dos percentuais de deságios oferecidos por cada empreendimento.

\section{CONSIDERAÇÕES FINAIS}

Este estudo trouxe duas contribuições teóricas à análise dos investimentos em energia eólica: identificação de fatores de sucesso obtidos unicamente com base em dados oriundos dos próprios fluxos de caixa dos projetos e descaracterização dos percentuais dos deságios oferecidos nos leilões como motivadores de sucesso ou fracasso nos empreendimentos.

Deste modo, este estudo atendeu ao objetivo a que se propôs ao responder à sua questão norteadora, e não refutou a hipótese levantada de que a dimensão de um projeto eólico é um fator determinante para o seu sucesso, inpendentemente do percentuaal de deságio oferecido.
Inicialmente, este estudo constatou que a volatilidade dos fluxos de caixa não era um fator que pudesse ser usado para auferir as possíveis diferenças entre os projetos eólicos bem-sucedidos e os que fracassaram, tendo em vista que todos os empreendimentos estavam sujeitos às mesmas incertezas e possuíam os mesmos pesos de custos fixos. Logo, todos teriam o mesmo risco, sob a ótica da teoria das opções reais.

Os fatores que estão mais associados a uma maior possibilidade de os projetos de usinas eólicas terem sucesso são que eles apresentem escores positivos da componente "Maiores Gerações e Maiores RiquezasMGMR", sendo essa caracterizada por investimentos mais significativos, maiores demandas de MWh e fluxos de caixa livre mais elevados.

Já com relação aos deságios oferecidos pelos investidores para que seus projetos se tornassem ganhadores nos certames que participaram, a componente MDES provou que os mesmos foram relevantes para causar atrasos na implementação das usinas eólicas, o que ratifica as assertivas de Azuela e Barroso (2012) e Del Rio e Linares (2014). Entretanto, o fato de um projeto estar em atraso em sua impletação não significa afirmar que o mesmo tenha mais ou menos propensão ao sucesso ou ao fracasso, sendo isso comprovado por meio de uma regressão logística.

A maior limitação encontrada durante a realização deste estudo refere-se à impossibilidade de acesso às informações alheias aos resultados nos LERs. Por exemplo, segundo dados de Pitombo (2019), foi devido a problemas de recuperação judicial enfrentados pela empresa Impsa, fornecedora de aerogeradores, que o parque eólico Casa Nova I, localizado no estado da Bahia, não conseguiu entrar em operação comercial, estando atualmente com nove anos de atraso com relação à data contratada. Ainda segundo o autor, a dificuldade de se procurar outro fabricante se deu porque cada um possui uma especificidade tecnologia diferente.

Como outra limitação, destaca-se a desconsideração do dólar neste estudo. Segundo Viana (2018), os aumentos constantes na taxa de câmbio é um dos principais fatores de atraso nos cronogramas de implantação dos projetos. Entretanto, não foi possível determinar a data exata que os materiais para fabricação da usina foram ou deveriam ser adquiridos para que se pudesse identificar qual o valor do dólar.

Para estudos futuros, sugere-se investigar se ocorreu alguma diferença nas características dos financiamentos obtidos pelos projetos aqui investigados, tendo em vista que os bem-sucedidos apresentaram dispêndios de capitais significativamente superiores aos que fracassaram. Além disso, a inclusão de variáveis alheias aos dados secundários dos leilões, bem como a utilização de outras metodologias para identificação dos fatores de sucesso, tais como as redes neurais, podem trazer informações que robusteçam a análise. 


\section{REFERÊNCIAS}

Agaton, C. B., \& Karl, H. (2018). A real options approach to renewable electricity generation in the Philippines. Energy, Sustainability and Society, 8(1), 1 http://dx.doi.org/10.1186/s13705-017-0143-y

Agência Nacional de Energia Elétrica. (2019). Informações do setor elétrico. http://www.aneel.gov.br

Amarante, O. A., Brower, M., \& Zack, J. (2001). Atlas do potencial eólico brasileiro. Brasília: Ministerio de Minas e Energia.

Assaf, A., Neto. (2014). Dividendos distribuídos no Brasil e no setor de energia elétrica. http://institutoassaf.com.br/wpcontent/uploads/2019/07/ANALISE DIV DIST 2014.pdf

Atalay, Y., Kalfagianni, A., \& Pattberg, P. (2017). Renewable energy support mechanisms in the Gulf Cooperation Council states: Analyzing the feasibility of feed-in tariffs and auction mechanisms. Renewable and Sustainable Energy Reviews, 72 723-733. http://dx.doi.org/10.1016/i.rser.2017.01.103

Azuela, G. E., \& Barroso, L. A. (2012). Design and performance of policy instruments to promote the development of renewable energy: emerging experience in selected developing countries. The World Bank. https://openknowledge.worldbank.org/bitstream/handle/10 986/9379/709090PUB0EPI0067869B09780821396025.pd f?sequence $=1$

Bayer, B., Berthold, L., \& de Freitas, B. M. R. (2018). The Brazilian experience with auctions for wind power: An assessment of project delays and potential mitigation measures. Energy policy, 122 http://dx.doi.org/10.1016/i.enpol.2018.07.004

97-117.

Bello, A. O., Oguntolu, F. A., Adetutu, O. M., \& Ojedokun, J. P. (2016). Application of Bootstrap Re-sampling Method to a Categorical Data of HIVIAIDS Spread across different Social-Economic Classes. arXiv preprint arXiv:1609.07806. http://dx.doi.org/10.5923/i.statistics.20150504.04

Bondarik, R., Pilatti, L. A., \& Horst, D. J. (2018). Uma visão geral sobre o potencial de geração de energias renováveis no brasil. Interciencia, 43(10), 680-688. https://www.redalyc.org/articulo.oa?id=33957861002

Bradshaw, A. (2018). Electricity Market Reforms and Renewable Energy: The Case of Wind and Solar in Brazil. Thesis (Doctor of Philosophy). Columbia University, Columbia, United States. https://academiccommons.columbia.edu/doi/10.7916/D8G B3MQT

Brandão, L. E., Dyer, J. S., \& Hahn, W. J. (2012). Volatility estimation for stochastic project value models. European Journal of Operational Research, 220(3), 642-648. http://dx.doi.org/10.1016/j.ejor.2012.01.059/

Câmara de Comercialização de Energia Elétrica. (2015). Infoleilão. 8o Leilão de Energia de Reserva. n. 16.18 nov. 2015. Recuperado

de

http://www.ccee.org.br/portal/faces/pages publico/o-quefazemos/infomercado?showFlag=F\& afrLoop $=619053533$ $\underline{003802}$

Campos, F. L. S., Ramos, F. L, \& Azevedo, B. M. (2016). Análise de viabilidade econômica regulatória à criação de cooperativa de consumo de energia elétrica: o caso do setor elétrico brasileiro na segunda década do século XXI. Revista Produção Online, 16(3), 966-987. http://dx.doi.org/10.14488/1676-1901.v16i3.2305

Carmo, C. R. S., Lima, A. D., Nunes, J. G. S., \& Saad, A. L. M. (2018). Uma análise acerca do perfil dos leilões de energia elétrica realizados em ambiente de contratação regulada de 2005 a 2016. Revista de Auditoria Governança e Contabilidade, $\quad 6 \quad$ (23), http://www.fucamp.edu.br/editora/index.php/ragc/issue/vie $\underline{w} / 91$
Castro, R. D. (2015). Energia solar térmica e fotovoltaica em residências: estudo comparativo em diversas localidades do Brasil. Dissertação (Mestrado em Planejamento e Sistemas Energéticos) - Programa de Pós-Graduação em Planejamento e Sistemas Energéticos. Universidade Estadual de Campinas, Campinas, São Paulo, Brasil. http://repositorio.unicamp.br/handle/REPOSIP/265829

Convênio ICMS 109, de 21 de outubro de 2014. Autoriza os Estados que menciona a conceder diferimento do ICMS devido nas operações com máquinas, equipamentos e materiais destinados à captação, geração e transmissão de energia elétrica ou eólica incorporados ao ativo imobilizado de estabelecimentos geradores de energia solar ou eólica. Recuperado de https://www.confaz.fazenda.gov.br/legislacao/convenios/2 $\underline{014 / C V 10914}$

Cuervo, F. I., \& Botero, S. B. (2016). Wind power reliability valuation in a Hydro-Dominated power market: The Colombian case. Renewable and Sustainable Energy Reviews, 57, 1359-1372. http://dx.doi.org10.1016/j.rser.2015.12.159/

Decreto n. 6.353, de 16 de janeiro de 2008. Regulamenta a contratação de energia de reserva. http://www.planalto.gov.br/ccivil 03/ Ato20072010/2008/Decreto/D6353.htm

Del Río, P., \& Linares, P. (2014). Back to the future? Rethinking auctions for renewable electricity support. Renewable and Sustainable Energy Reviews, 35, 42-56. http://dx.doi.org/10.1016/j.rser.2014.03.03

Empresa de Pesquisas Energéticas. (2017). Plano nacional de expansão de energia elétrica 2026. http://www.epe.gov.br/pt/publicacoes-dadosabertos/publicacoes/plano-decenal-de-expansao-deenergia-pde

Ferreira, H. L., Patah, L. A., \& Faria, R. M. (2017). Winner's curse: evidências da maldição do vencedor entre empreendimentos eólicos e fotovoltaicos vencedores do $8^{\circ}$ leilão de energia de reserva. Anais do Simpósio Internacional de Gestão de Projetos, Inovação e Sustentabilidade, São Paulo, São Paulo, Brasil. https://www.researchgate.net/profile/Heitor Ferreira2/publi cation/321003264 WINNER\%27S CURSE EVIDENCIAS DA MALDICAO DO VENCEDOR ENTRE EMPREEN DIMENTO EOLICOS E FOTOVOLTAICOS VENCEDO RES DO 8 LEILAO DE ENERGIA RESERVA/links/5a0 670884585157013a3c252/WINNERS-CURSEEVIDENCIAS-DA-MALDICAO-DO-VENCEDOR-ENTREEMPREENDIMENTO-EOLICOS-E-FOTOVOLTAICOSVENCEDORES-DO-8-LEILAO-DE-ENERGIARESERVA.pdf

Fontanet, F.A. (2012) Avaliação de uma opção de espera de um parque eólico pelo método das opções reais. Dissertação (Mestrado em Engenharia Elétrica), Pontifícia Universidade Católica do Rio de Janeiro, Rio de Janeiro, Brasil. https://www.maxwell.vrac.pucrio.br/colecao.php?strSecao=resultado\&nrSeq=22813@1

González, A., Riba, J. R., Rius, A., \& Puig, R. (2015). Optimal sizing of a hybrid grid-connected photovoltaic and wind power system. Applied Energy, 154, 752-762. http://dx.doi.org/10.1016/j.apenergy.2015.04.105

Hair, J. F., Jr., Black, W.C., Babin, B.J., \& Anderson, R. E. (2010). Multivariate Data Analysis. New Jersey: Pearson Prentice Hall.

Hochberg, M., \& Poudineh, R. (2018). Renewable Auction Design in Theory and Practice: Lessons from the Experience of Brazil and Mexico. Oxford: Oxford Institute for Energy Studies.

Hongyu, K., Sandanielo, V. L. M., \& Oliveira, G. J., Júnior. (2016). Análise de componentes principais: resumo teórico, aplicação e interpretação. E\&S Engineering and 
Science, 5(1), 83-90.

http://dx.doi.org/10.18607/ES20165053

Jolliffe, I. T., \& Cadima, J. (2016). Principal component analysis: a review and recent developments. Philosophical Transactions of the Royal Society A: Mathematical, Physical and Engineering Sciences, 374(2065), 20150202. http://dx.doi.org/10.1098/rsta.2015.0202

Kaiser, H. F. (1974). An index of factorial simplicity. Psychometrika, 39(1), 31-36. https://link.springer.com/article/10.1007/BF02291575

Ketsiri, K. (2012). Weather exposure and market price of weather risk. Thesis (Doctorate Degree's in Philosophy in Finance). University of Exeter, United King. https://eric.exeter.ac.uk/repository/handle/10036/3904

Khanna, D., Sahu, R., Baths, V., \& Deshpande, B. (2015). Comparative study of classification techniques (SVM, logistic regression and neural networks) to predict the prevalence of heart disease. International Journal of Machine Learning and Computing, 5(5), 414. http://dx.doi.org/10.7763/IJMLC.2015.V5.544

Kim, D., Chi, S., \& Kim, J. (2018). Selecting network-level project sections for sustainable pavement management in Texas. Sustainability, $\quad 10(3), \quad 686$. http://dx.doi.org/10.3390/su10030686.

Klieštik, T., Kočišová, K., \& Mišanková, M. (2015). Logit and probit model used for prediction of financial health of company. Procedia economics and finance, 23, 850-855. http://dx.doi.org/ 10.1016/S2212-5671(15)00485-2

Kreiss, J., Ehrhart, K. M., \& Haufe, M. C. (2017). Appropriate design of auctions for renewable energy supportPrequalifications and penalties. Energy Policy, 101, 512520. http://dx.doi.org/ 10.1016/j.enpol.2016.11.007

Kuhn, M., \& Johnson, K. (2013). Applied predictive modeling (Vol. 26). New York: Springer.

Lai, K. H., Cheng, T. E., \& Yeung, A. C. (2004). An empirical taxonomy for logistics service providers. Maritime Economics \& Logistics, 6(3), 199-219. http://dx.doi.org/ 10.1057/palgrave.mel.9100109

Lakatos, E. M, \& Marconi, M. A. (2010). Fundamentos de metodologia científica: Técnicas de pesquisa. 7. ed. São Paulo: Atlas.

Lee, G. (Ed.). (2014). E-Commerce, E-Business and E-Service. Boca Raton: CRC Press.

Lei n. 11.488, de 15 de junho de 2007. Cria o regime especial de incentivos para o desenvolvimento da infra-estruturaREIDI. Recuperado http://www.planalto.gov.br/ccivil 03/ Ato20072010/2007/Lei/L11488.htm

Lei n. 12.783 , de 11 de janeiro de 2013. Dispõe sobre as concessões de geração, transmissão e distribuição de energia elétrica e dá outras providências. Recuperado de http://www.planalto.gov.br/ccivil 03/ Ato20112014/2013/Lei/L12783.htm

Lindemeyer, R. M. (2018). Aplicação da teoria de opções reais na avaliação de um complexo eólico. Dissertação (Mestrado em Economia). Escola de Economia de São Paulo. Fundação Getúlio Vargas, São Paulo, São Paulo, Brasil. https://bibliotecadigital.fgv.br/dspace/handle/10438/24787

Maskey, R., Fei, J., \& Nguyen, H. O. (2018). Use of exploratory factor analysis in maritime research. The Asian journal of shipping and logistics, 34(2), 91-111. http://dx.doi.org/ 10.1016/j.ajsl.2018.06.006

McFadden, D. (1977). Quantitative methods for analyzing travel behavior of individuals: some recent developments. Berkeley: Cowles Foundation Discussion Papers 474, Cowles Foundation for Research in Economics, Yale University. https://ideas.repec.org/p/cwl/cwldpp/474.html
Monteiro, N. D. S. C., Monteiro, R. A. B., Mariano, J. D. A., Urbanetz, J., Jr., \& Romano, C. A. (2017). Brazil Market Outlook for Photovoltaic Solar Energy: A Survey Study. Current Journal of Applied Science and Technology, 1-11. http://dx.doi.org/ 10.9734/BJAST/2017/33408

Naik, G. R. (2017). Advances in Principal Component Analysis: Research and Development. Zug: Springer.

Nakabayashi, R. K. (2014). Microgeração fotovoltaica no Brasil: condições atuais e perspectivas futuras. Dissertação (Mestrado em Energia) - Instituto de Energia e Ambiente, Universidade de São Paulo, São Paulo, São Paulo, Brasil, 2014.

https://teses.usp.br/teses/disponiveis/106/106131/tde26012015-141237/en.php

Nascimento, R. L. (2017). Energia solar no Brasil: situação e perspectivas.

http://bd.camara.gov.br/bd/handle/bdcamara/32259

Oliveira, E. M, \& Oliveira, F. L. C. (2017). Padrões de variabilidade em vazões afluentes a usinas hidrelétricas e associações com massas de ar. Cadernos do IME-Série Estatística, 43(1),

http://dx.doi.org/10.12957/cadest.2017.31363

18-38.

Operador Nacional do Sistema do Sistema Elétrico. (2019). Boletim mensal de geração de energia. http://www.ons.org.br/Paginas/busca.aspx?k=boletim.

Pereira, F. P., Jr. (2018). Teoria das opções reais para avaliar investimento sem disposição de rejeitos com menor impacto socioambiental: uma análise comparativa com a metodologia do fluxo de caixa descontado na Samarco Minerado S/A. Dissertação (Mestrado em Administração). Fundação Dom Cabral, Belo Horizonte, Minas Gerais, Brasil.

http://acervo.ci.fdc.org.br/AcervoDigital/Disserta\%C3\%A7 \%C3\%B5es\%20FDC/MPA\%202018/Disserta\%C3\%A7\%C 3\%A30\%20Francisco\%20de\%20Paula\%20Pereira\%20Ju nior.pdf

Pinto, L. I. C., Martins, F. R., \& Pereira, E. B. (2017). O mercado brasileiro da energia eólica, impactos sociais e ambientais. Ambiente \& Água-An Interdisciplinary Journal of Applied Science, 12(6), 1082-1100. http://dx.doi.org/ 10.4136/1980-993X

Pitombo, J. P. (2019). Nove anos após leilão, torres eólicas estão abandonadas na Bahia. Jornal Folha de São Paulo. https://www1.folha.uol.com.br/mercado/2019/10/noveanos-apos-leilao-torres-eolicas-estao-abandonadas-nabahia.shtml

Resolução Normativa nํ 608, de 25 de março de 2014. Aprova o Submódulo 12.3 dos procedimentos de regularização tarifária-PRORET, e dá outras providências. Recuperado de

http://www2.aneel.gov.br/aplicacoes/audiencia/arquivo/20 14/002/resultado/resolucao normativa n\%C2\%BA 608, de 25 de marco de 2014..pdf

Resolução Normativa no 780, de 25 de julho de 2017. Estabelece critérios para o Operador Nacional do Sistema desempenhar as atividades de gestão orçamentária, e dá outras providências. Recuperado de http://www2.aneel.gov.br/aplicacoes/audiencia/dspListaRe sultado.cfm?attAnoAud=2013\&attldeAud=753\&attAnoFas Aud=2013\&id area $=13$

Ribeiro, S. S., \& Silva, A. F. A., Jr. (2016). Avaliação de Políticas Promovidas pela ANEEL para Incentivo da Geração de Energia Elétrica por Fonte Solar. Anais do Encontro da Associação dos Programas de pós-Graduação em Administração. Costa do Sauipe, Bahia, Brasil. https://www.researchgate.net/publication/308796506 Aval iacao de Politicas Promovidas pela ANEEL para Ince ntivo da Geracao de Energia Eletrica por Fonte Solar

Rodrigues, L. B., Perobelli, F. F. C., \& Vasconcelos, S. (2017). Geração de energia eólica no Brasil: um investimento 
viável?. Revista Brasileira de Economia de

Empresas, $17(2)$.

https://portalrevistas.ucb.br/index.php/rbee/article/view/81

$\underline{96}$

Rossi, R. G. (2017). Análise dos componentes principais em Data Warehouses. Dissertação (Mestrado em Ciências). Instituto de Matemática e Estatística. Universidade de São Paulo, São Paulo, São Paulo, Brasil. https://teses.usp.br/teses/disponiveis/45/45134/tde07012018-182730/publico/mestrado.pdf

Sawin, J. (2017). Renewable energy policy network for the 21st century renewables 2017 global status report. REN21
Secretariat: Paris, France, 1-302. http://gesel.ie.ufrj.br/app/webroot/files/IFES/BV/ren213.pdf

Silva, A. F. A., Jr., \& Ribeiro, S. S. (2016). Avaliação de Leilões de Energia Solar Utilizando Opções Reais. Anais do Congresso Brasileiro de Planejamento Estratégico CBPE, Gramado, Rio Grande do Sul, Brasil, 10. https://www.researchgate.net/publication/308796830 Aval iacao de Leiloes de Energia Solar Utilizando Opcoes $\underline{\text { Reais }}$

Silva, A. F. A., Jr., Ribeiro, S. S., \& Quintella, V. M. (2018). Evaluation of Brazilian Auctions for Photovoltaic Projects Using Traditional and Real Option Approaches. SSRN Electronic Journal. http://dx.doi.org/10.2139/ssrn.3131964

Soares, T. M. C., \& Campos, C. P. (2018). Energia eólica na Bahia: Seus parques e sua contribuição para a matriz energética do estado. Cadernos de Prospecção, 11(5), 1318. http://dx.doi.org/ 10.9771/cp.v12i5.27396

Spratt, H., Ju, H., \& Brasier, A. R. (2013). A structured approach to predictive modeling of a two-class problem using multidimensional data sets. Methods, 61(1), 73-85. http://dx.doi.org/ 10.1016/j.ymeth.2013.01.002

Sur, P., \& Candès, E. J. (2018). A modern maximum-likelihood theory for high-dimensional logistic regression. arXiv preprint http://dx.doi.org/10.1073/pnas.1810420116

Torinelli, V. H., Silva, A. F.A. Jr., \& Andrade, J. C. S. (2018). Wind power energy in Brazil: public financing and future perspectives. Latin American Journal of Management for Sustainable Development, 4(1), 41-54. https://www.researchgate.net/profile/Jose Andrade52/pub/ ication/324845781 Wind power energy in Brazil public financing and future perspectives/links/5bcf1203299bf1 a43d9a790a/Wind-power-energy-in-Brazil-publicfinancing-and-future-perspectives.pdf

Vargas, A., Saavedra, O. R., Samper, M. E., Rivera, S., \& Rodriguez, R. (2016). Latin american energy markets: Investment opportunities in nonconventional renewables. IEEE Power and Energy Magazine, 14(5), 3847. http://dx.doi.org/10.1109/MPE.2016.2573862

Viana, A. G. (2018). Leilões como mecanismo alocativo para um novo desenho de mercado no Brasil. Tese (Doutorado em Ciências) - Escola Politécnica da Universidade de São Paulo, Universidade de São Paulo, São Paulo, São Paulo, Brasil. https://www.teses.usp.br/teses/disponiveis/3/3143/tde06042018-082743/pt-br.php

Zabaloy, M. F., \& Guzowski, C. (2018). Energy transition policy from fossil fuels to renewable energy: the case of Argentina, Brazil and Uruguay in 1970-2016 period. Economía Coyuntural, 3, 02. https://www.researchgate.net/publication/334170790 ENE RGY TRANSITION POLICY FROM FOSSIL FUELS T O RENEWABLE ENERGY THE CASE OF ARGENTIN A BRAZIL AND URUGUAY IN 19702016 PERIOD 


\section{CONTEXTUS}

REVISTA CONTEMPORÂNEA DE ECONOMIA E GESTÃO

\section{CONTEXTUS}

REVISTA CONTEMPORÂNEA

DE ECONOMIA E GESTÃO

\section{FACULDADE DE ECONOMIA, ADMINISTRAÇÃO, ATUÁRIA E CONTABILIDADE (FEAAC)}

Av. da Universidade - 2486, Benfica

CEP 60020-180, Fortaleza-CE

DIRETORIA: Paulo Rogério Faustino Matos Danielle Augusto Peres

Website: www.periodicos.ufc.br/contextus

E-mail: revistacontextus@gmail.com

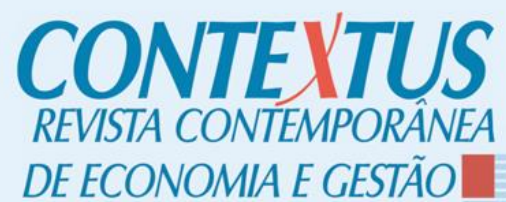

UNIVERSIDADE

FEDERAL

DO CEARÁ

FACULDADE

DE ECONOMIA,

ADMINISTRAČÃO,

ATUÁRIA

E CONTABILIDADE

\section{CONTEXTUS}

REVISTA CONTEMPORÂNEA DE ECONOMIA E GESTÃO.

\section{ISSN 1678-2089}

\section{ISSNe 2178-9258}

1. Economia, Administração e Contabilidade - Periódico

2. Universidade Federal do Ceará. FEAAC - Faculdade de

Economia, Administração, Atuária e Contabilidade

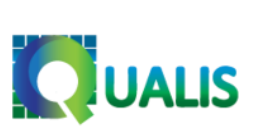

A Contextus está classificada no sistema Qualis - Capes como periódico B1, na área de Administração Pública e de Empresas, Ciências Contábeis e Turismo (2013-2016).

A Contextus está de acordo e assina a Avaliação de Pesquisas (DORA).

\section{EDITOR-CHEFE}

Diego de Queiroz Machado (UFC)

\section{EDITORAS ADJUNTAS}

Alane Siqueira Rocha (UFC)

Márcia Zabdiele Moreira (UFC)

\section{EDITORES ASSOCIADOS}

Alessandra de Sá Mello da Costa (IAG/PUC-Rio)

Andrew Beheregarai Finger (UFAL)

Armindo dos Santos de Sousa Teodósio (PUC-MG)

Carlos Enrique Carrasco Gutierrez (UCB)

Gabriel Moreira Campos (UFES)

Guilherme Jonas Costa da Silva (UFU)

Henrique César Muzzio de Paiva Barroso (UFPE)

Jorge de Souza Bispo (UFBA)

Keysa Manuela Cunha de Mascena (UNIFOR)

Minelle Enéas da Silva (La Rochelle, França)

Rafael Fernandes de Mesquita (IFPI)

Rosimeire Pimentel (UFES)

Susana Jorge (UC, Portugal)

Thiago Henrique Moreira Goes (UFPR)

\section{CONSELHO EDITORIAL}

Ana Sílvia Rocha Ipiranga (UECE)

Conceição de Maria Pinheiro Barros (UFC)

Danielle Augusto Peres (UFC)

Diego de Queiroz Machado (UFC)

Editinete André da Rocha Garcia (UFC)

Emerson Luís Lemos Marinho (UFC)

Eveline Barbosa Silva Carvalho (UFC)

Fátima Regina Ney Matos (ISMT)

Mario Henrique Ogasavara (ESPM)

Paulo Rogério Faustino Matos (UFC)

Rodrigo Bandeira-de-Mello (FGV-EAESP)

Vasco Almeida (ISMT)

\section{CORPO EDITORIAL CIENTÍFICO}

Alexandre Reis Graeml (UTFPR)

Augusto Cezar de Aquino Cabral (UFC)

Denise Del Pra Netto Machado (FURB)

Ednilson Bernardes (Georgia Southern University)

Ely Laureano Paiva (FGV-EAESP)

Eugenio Ávila Pedrozo (UFRGS)

Francisco José da Costa (UFPB)

Isak Kruglianskas (FEA-USP)

José Antônio Puppim de Oliveira (UCL)

José Carlos Barbieri (FGV-EAESP)

José Carlos Lázaro da Silva Filho (UFC)

José Célio de Andrade (UFBA)

Luciana Marques Vieira (UNISINOS)

Luciano Barin-Cruz (HEC Montréal)

Luis Carlos Di Serio (FGV-EAESP)

Marcelle Colares Oliveira (UFC)

Maria Ceci Araujo Misoczky (UFRGS)

Mônica Cavalcanti Sá Abreu (UFC)

Mozar José de Brito (UFL)

Renata Giovinazzo Spers (FEA-USP)

Sandra Maria dos Santos (UFC)

Walter Bataglia (MACKENZIE)
ABEC Brash
A Contextus é associada à Associação Brasileira de Editores Científicos (ABEC).

Esta obra está licenciada com uma licença Creative Commons Atribuição - Não Comercial 4.0 Internacional. 